Pure and Applied Mathematics Quarterly

Volume 2, Number 3

(Special Issue: In honor of

Robert MacPherson, Part 1 of 3)

$655-671,2006$

\title{
Nested Complexes and their Polyhedral Realizations
}

\author{
Andrei Zelevinsky
}

To Bob MacPherson on the occasion of his 60th birthday

\section{INTRODUCTION}

This note which can be viewed as a complement to [9], presents a self-contained overview of basic properties of nested complexes and their two dual polyhedral realizations: as complete simplicial fans, and as simple polytopes. Most of the results are not new; our aim is to bring into focus a striking similarity between nested complexes and associated fans and polytopes on one side, and cluster complexes and generalized associahedra introduced and studied in $[7,2]$ on the other side.

First a very brief history (more details and references can be found in $[10,9]$ ). Nested complexes appeared in the work by De Concini - Procesi [3] in the context of subspace arrangements. More general complexes associated with arbitrary finite graphs were introduced and studied in [1] and more recently in [10]; these papers also present a construction of associated simple convex polytopes (dubbed graph associahedra in [1], and De Concini - Procesi associahedra in [10]). An even more general setup developed by Feichtner - Yuzvinsky in [6] associates a nested complex and a simplicial fan to an arbitrary finite atomic lattice. In the present note we follow [5] and [9] in adopting an intermediate level of generality, and studying the nested complexes associated to building sets (see Definition 2.1 below).

Feichtner and Sturmfels in [5] show that the simplicial fan associated to a building set is the normal fan of a simple convex polytope, while Postnikov in [9] gives an elegant construction of this polytope as a Minkowski sum of simplices.

Received July 29, 2005.

2000 Mathematics Subject Classification.Primary 52B.

Research supported by NSF grant DMS-0500534. 
In this note we develop an alternative approach, constructing the polytope directly from the fan; this construction is completely analogous to that in [2]. The main role in this approach is played by the link decomposition property (Proposition 3.2 below) asserting that the link of any element in a nested complex can be naturally identified with a nested complex of smaller rank. This result provides a unified method for establishing properties of nested complexes and their polyhedral realizations by induction on the rank of a building set.

The note is structured as follows. Section 2 introduces (basically following [9]) building sets and associated nested complexes. Section 3 presents the link decomposition property (Proposition 3.2). In this generality it seems to be new; for the graph associahedra it appeared in [10]. Section 4 contains some structural properties of nested complexes, all of them derived in a unified way from Proposition 3.2.

In Section 5 we show that any nested complex can be realized as a smooth complete simplicial fan (Theorem 5.1 and Corollary 5.2); we refer to this fan as the nested fan. This construction has appeared in $[6,4,5]$. However, it is not so easy to extract from these papers a proof that the nested fan is well-defined; this is why we prefer to give a simple self-contained proof.

In Section 6, we prove (Theorem 6.1) that the nested fan is the normal fan of a simple polytope $\Pi$, which we call the nested polytope. The argument is completely parallel to that in [2]; in particular, it leads to a specific realization of $\Pi$ which can be shown to be the same as the realization given in [9].

The concluding Section 7 discusses the special class of graphical building sets, those giving rise to graph associahedra $[1,10]$. We give a simple characterization of graphical building sets (Proposition 7.3). For them an analogy with cluster complexes becomes sharper: in particular, the corresponding nested complex is a clique complex (Corollary 7.4).

\section{NeSted COMPLEXES}

We start by recalling basic definitions from [9, Section 7].

Definition 2.1 ([9], Definition 7.1). Let $S$ be a nonempty finite set. A building set (or simply a building) on $S$ is a collection $\mathcal{B}$ of nonempty subsets of $S$ satisfying the conditions:

(B1) If $I, J \in \mathcal{B}$ and $I \cap J \neq \emptyset$, then $I \cup J \in \mathcal{B}$.

(B2) $\mathcal{B}$ contains all singletons $\{i\}$, for $i \in S$.

The nested complex associated to a building $\mathcal{B}$ is defined as follows. Let $\mathcal{B}_{\max } \subset$ $\mathcal{B}$ be the set of maximal (by inclusion) elements of $\mathcal{B}$. We refer to the elements of $\mathcal{B}_{\max }$ as the $\mathcal{B}$-components. In view of (B1) and (B2), the $\mathcal{B}$-components 
are pairwise disjoint, and their union is $S$. We define the rank of $\mathcal{B}$ by setting $\operatorname{rk}(\mathcal{B})=|S|-\left|\mathcal{B}_{\max }\right|$.

Definition 2.2 (cf. [9], Definition 7.3). A subset $N \subset \mathcal{B}-\mathcal{B}_{\max }$ is called nested if it satisfies the following condition: for any $k \geq 2$ and any $I_{1}, \ldots, I_{k} \in N$ such that none of the $I_{i}$ contains another one, the union $I_{1} \cup \cdots \cup I_{k}$ is not in $\mathcal{B}$. The simplicial complex on $\mathcal{B}-\mathcal{B}_{\max }$ formed by the nested sets is called the nested complex and denoted $\mathcal{N}(\mathcal{B})$.

In particular, the 1-simplices of $\mathcal{N}(\mathcal{B})$ are pairs $\{I, J\} \subset \mathcal{B}$ such that:

$$
\text { either } I \subset J \text {, or } J \subset I \text {, or } I \cup J \notin \mathcal{B}
$$

(in view of (B2), in the last case $I$ and $J$ are disjoint). Note that our definition of nested sets is different from the one in [9, Definition 7.3]: Postnikov's nested sets are obtained from ours by adjoining the set $\mathcal{B}_{\max }$. Of course this does not affect the poset structure of the nested complex; an advantage of the present version is that our nested sets form a simplicial complex.

\section{LINK DECOMPOSITION}

Let $\mathcal{B}$ be a building on $S$, and $\mathcal{N}(\mathcal{B})$ the associated nested complex. For every $C \in \mathcal{B}-\mathcal{B}_{\text {max }}$, let

$$
\mathcal{N}(\mathcal{B})_{C}=\left\{N^{\prime} \subset \mathcal{B}-\mathcal{B}_{\max }-\{C\}: N^{\prime} \cup\{C\} \in \mathcal{N}(\mathcal{B})\right\}
$$

denote the link of $C$ in $\mathcal{N}(\mathcal{B})$; this is a simplicial complex on the set $\{I \in$ $\left.\mathcal{B}-\mathcal{B}_{\max }-\{C\}:\{C, I\} \in \mathcal{N}(\mathcal{B})\right\}$. Our main tool in studying the structure of $\mathcal{N}(\mathcal{B})$ is the recursive description of $\mathcal{N}(\mathcal{B})_{C}$ given in Proposition 3.2 below. To state it, we need some preparation.

Definition 3.1. Let $C$ be a nonempty subset of $S$.

- The restriction of $\mathcal{B}$ to $C$ is the building on $C$ defined by

$$
\left.\mathcal{B}\right|_{C}=\{I \subset C: I \in \mathcal{B}\} .
$$

- The contraction of $C$ from $\mathcal{B}$ is the building on $S-C$ defined by

$$
C \backslash \mathcal{B}=\{I \subset S-C: I \in \mathcal{B} \text { or } C \cup I \in \mathcal{B}\} .
$$

The fact that both $\left.\mathcal{B}\right|_{C}$ and $C \backslash \mathcal{B}$ are indeed buildings, is immediate from Definition 2.1.

Let $S_{1}, \ldots, S_{k}$ be disjoint finite sets, and let $\mathcal{B}_{i}$ be a building on $S_{i}$ for $i=$ $1, \ldots, k$. The product $\mathcal{B}_{1} \times \cdots \times \mathcal{B}_{k}$ is defined as the building on $S_{1} \sqcup \cdots \sqcup S_{k}$ formed by the elements of all the $\mathcal{B}_{i}$. Clearly, the nested complex $\mathcal{N}\left(\mathcal{B}_{1} \times \cdots \times \mathcal{B}_{k}\right)$ is naturally isomorphic to the product $\mathcal{N}\left(\mathcal{B}_{1}\right) \times \cdots \times \mathcal{N}\left(\mathcal{B}_{k}\right)$. In particular, every building $\mathcal{B}$ on $S$ decomposes as a product $\left.\mathcal{B}\right|_{S_{1}} \times \cdots \times\left.\mathcal{B}\right|_{S_{k}}$, where $S_{1}, \ldots, S_{k}$ are the $\mathcal{B}$-components; therefore, $\mathcal{N}(\mathcal{B})$ can be identified with $\mathcal{N}\left(\left.\mathcal{B}\right|_{S_{1}}\right) \times \cdots \times \mathcal{N}\left(\left.\mathcal{B}\right|_{S_{k}}\right)$. 
Proposition 3.2 (Link Decomposition). For every $C \in \mathcal{B}-\mathcal{B}_{\max }$, the link $\mathcal{N}(\mathcal{B})_{C}$ is isomorphic to $\mathcal{N}\left(\left.\mathcal{B}\right|_{C} \times C \backslash \mathcal{B}\right)$.

Proof. Fix $C \in \mathcal{B}-\mathcal{B}_{\max }$ and abbreviate $\mathcal{B}^{\prime}=\left.\mathcal{B}\right|_{C} \times C \backslash \mathcal{B}$. Let

$$
\mathcal{B}_{0}=\left\{I \in \mathcal{B}-\mathcal{B}_{\max }-\{C\}:\{C, I\} \in \mathcal{N}(\mathcal{B})\right\}
$$

be the ground set of $\mathcal{N}(\mathcal{B})_{C}$. In view of $(2.1), \mathcal{B}_{0}$ is the disjoint union of the following three sets:

$$
\begin{aligned}
& \mathcal{B}_{1}=\{I \in \mathcal{B}-\{C\}: I \subset C\} ; \\
& \mathcal{B}_{2}=\left\{I \in \mathcal{B}-\mathcal{B}_{\text {max }}-\{C\}: C \subset I\right\} ; \\
& \mathcal{B}_{3}=\left\{I \in \mathcal{B}-\mathcal{B}_{\text {max }}: C \cap I=\emptyset, C \cup I \notin \mathcal{B}\right\} .
\end{aligned}
$$

Now to every $I \in \mathcal{B}_{0}=\mathcal{B}_{1} \cup \mathcal{B}_{2} \cup \mathcal{B}_{3}$ we associate a subset $I^{\prime} \subset S$ by setting

$$
I^{\prime}= \begin{cases}I & \text { if } I \in \mathcal{B}_{1} \cup \mathcal{B}_{3} ; \\ I-C & \text { if } I \in \mathcal{B}_{2} .\end{cases}
$$

Remembering the definitions, we see that $\mathcal{B}_{1}$ is the ground set of the nested complex $\mathcal{N}\left(\left.\mathcal{B}\right|_{C}\right)$, and $\mathcal{B}_{3}$ is a subset of the ground set of $\mathcal{N}(C \backslash \mathcal{B})$ formed by those $I^{\prime} \subset S-C$ for which $C \cup I^{\prime} \notin \mathcal{B}$. Furthermore, the correspondence $I \mapsto I^{\prime}=I-C$ identifies $\mathcal{B}_{2}$ with the remaining part of the ground set of $\mathcal{N}(C \backslash \mathcal{B})$, formed by those $I^{\prime} \subset S-C$ for which $C \cup I^{\prime} \in \mathcal{B}$. Thus, the correspondence $I \mapsto I^{\prime}$ is a bijection between the ground sets of $\mathcal{N}(\mathcal{B})_{C}$ and $\mathcal{N}\left(\mathcal{B}^{\prime}\right)$. To prove Proposition 3.2, it remains to show the following:

The correspondence $I \mapsto I^{\prime}$ induces an

$$
\text { isomorphism of complexes } \mathcal{N}(\mathcal{B})_{C} \text { and } \mathcal{N}\left(\mathcal{B}^{\prime}\right) \text {. }
$$

Thus, we need to show the following: for any distinct $I_{1}, \ldots, I_{k} \in \mathcal{B}_{0}$, the set $\left\{C, I_{1}, \ldots, I_{k}\right\}$ is nested for $\mathcal{B}$ if and only if $\left\{I_{1}^{\prime}, \ldots, I_{k}^{\prime}\right\}$ is nested for $\mathcal{B}^{\prime}$. First suppose $\left\{I_{1}^{\prime}, \ldots, I_{k}^{\prime}\right\}$ is not nested for $\mathcal{B}^{\prime}$. Without loss of generality, we can assume that $k \geq 2$, none of the $I_{i}^{\prime}$ contains another one, and $I_{1}^{\prime} \cup \cdots \cup$ $I_{k}^{\prime} \in \mathcal{B}^{\prime}$. Choosing the smallest possible $k$ with this property and using property (B1) in Definition 2.1, we can further assume that the $I_{i}^{\prime}$ are pairwise disjoint. Remembering the definition of $\mathcal{B}^{\prime}$, we see that either all the $I_{i}^{\prime}$ are contained in $C$, or all the $I_{i}^{\prime}$ are contained in $S-C$. In the former case, $I_{i}=I_{i}^{\prime}$ for all $i$, so we have $I_{1} \cup \cdots \cup I_{k} \in \mathcal{B}$, implying that $\left\{C, I_{1}, \ldots, I_{k}\right\}$ is not nested for $\mathcal{B}$. Now suppose that all the $I_{i}^{\prime}$ are contained in $S-C$. Remembering (3.3), we see that the condition $I_{1}^{\prime} \cup \cdots \cup I_{k}^{\prime} \in \mathcal{B}^{\prime}$ means that at least one of the subsets $I_{1}^{\prime} \cup \cdots \cup I_{k}^{\prime}$ and $C \cup I_{1}^{\prime} \cup \cdots \cup I_{k}^{\prime}$ belongs to $\mathcal{B}$. If $I_{i}^{\prime}=I_{i}$ for all $i=1, \ldots, k$, we again conclude that $\left\{C, I_{1}, \ldots, I_{k}\right\}$ is not nested for $\mathcal{B}$. Therefore suppose that $I_{i}=C \cup I_{i}^{\prime}$ for some $i$. If $I_{j}=C \cup I_{j}^{\prime}$ for some $i \neq j$, then $I_{i} \cup I_{j} \in \mathcal{B}$ by property (B1) in Definition 2.1, and so $\left\{C, I_{1}, \ldots, I_{k}\right\}$ is not nested for $\mathcal{B}$ in this case as 
well. Finally, if $I_{j}=I_{j}^{\prime}$ for $j \neq i$, then $I_{1}, \ldots, I_{k}$ are pairwise disjoint, and their union belongs to $\mathcal{B}$, again implying that $\left\{C, I_{1}, \ldots, I_{k}\right\}$ is not nested for $\mathcal{B}$.

The converse implication (if $\left\{C, I_{1}, \ldots, I_{k}\right\}$ is not nested for $\mathcal{B}$ then $\left\{I_{1}^{\prime}, \ldots, I_{k}^{\prime}\right\}$ is not nested for $\mathcal{B}^{\prime}$ ) follows by reversing the above arguments.

\section{Some APPLiCATIONS OF THE LINK DECOMPOSITION}

Proposition 4.1. The nested complex $\mathcal{N}(\mathcal{B})$ is pure of dimension $\operatorname{rk}(\mathcal{B})-1$, that is, all maximal nested sets are of the same cardinality $\operatorname{rk}(\mathcal{B})$.

Proof. Proceed by induction on $\operatorname{rk}(\mathcal{B})$. The statement is trivial if $\operatorname{rk}(\mathcal{B})=0$ : in this case, $\mathcal{B}$ consists of the singletons, and $\mathcal{N}(\mathcal{B})$ is just empty. Now assume that $\operatorname{rk}(\mathcal{B}) \geq 1$, and let $N \subset \mathcal{B}-\mathcal{B}_{\max }$ be a maximal nested set. Let $C \in N$. Inspecting Definition 3.1, we see that $\left|\left(\left.\mathcal{B}\right|_{C} \times C \backslash \mathcal{B}\right)_{\max }\right|=\left|\mathcal{B}_{\max }\right|+1$ (the $\mathcal{B}$-components that are disjoint from $C$ remain intact, while the component $S^{\prime}$ containing $C$ splits into the two $\left(\left.\mathcal{B}\right|_{C} \times C \backslash \mathcal{B}\right)$-components $C$ and $\left.S^{\prime}-C\right)$. Thus, $\operatorname{rk}\left(\left.\mathcal{B}\right|_{C} \times C \backslash \mathcal{B}\right)=\operatorname{rk}(\mathcal{B})-1$. Using Proposition 3.2 and the induction assumption, we conclude that

$$
|N|=1+\operatorname{rk}\left(\left.\mathcal{B}\right|_{C} \times C \backslash \mathcal{B}\right)=\operatorname{rk}(\mathcal{B}),
$$

as claimed.

Proposition 4.2. For every maximal nested set $N$ and every $I \in N$, there is precisely one maximal nested set $N^{\prime}$ such that $N \cap N^{\prime}=N-\{I\}$.

Proof. First assume that $\operatorname{rk}(\mathcal{B})=1$. Then $\mathcal{B}$ consists of all singletons in $S$ and just one two-element set $\{i, j\}$. Thus, the only two maximal nested sets are $N=\{I\}$ and $N^{\prime}=\{J\}$, where $I$ (resp. $J$ ) is the singleton $\{i\}$ (resp. $\{j\}$ ). So our assertion holds.

Now let $\operatorname{rk}(\mathcal{B})>1$. Choose any $C \in N-\{I\}$. To prove Proposition 4.2, it suffices to show that the assertion holds for the link $\mathcal{N}(\mathcal{B})_{C}$. It remains to apply Proposition 3.2 and induction on $\operatorname{rk}(\mathcal{B})$.

Definition 4.3. The dual graph of the nested complex $\mathcal{N}(\mathcal{B})$ has maximal nested sets as vertices, with $N$ and $N^{\prime}$ joined by an edge whenever $\left|N \cap N^{\prime}\right|=|N|-1$.

The following property is immediate from Proposition 4.2.

Proposition 4.4. The dual graph of $\mathcal{N}(\mathcal{B})$ is regular of degree $\operatorname{rk}(\mathcal{B})$.

We now give a more detailed description of the edges of the dual graph.

Proposition 4.5. Let $N_{1}$ and $N_{2}$ be two maximal nested sets in $\mathcal{N}(\mathcal{B})$ joined by an edge in the dual graph, and let $N_{1} \cap N_{2}=N_{1}-\left\{I_{1}\right\}=N_{2}-\left\{I_{2}\right\}$. Then the following properties hold: 
(1) Neither of $I_{1}$ and $I_{2}$ contains another one.

(2) If $I_{1} \cap I_{2} \neq \emptyset$ then all the $\left.\mathcal{B}\right|_{I_{1} \cap I_{2}}$-components belong to $N_{1} \cap N_{2}$.

(3) There exist pairwise disjoint $I_{3}, \ldots, I_{k} \in N_{1} \cap N_{2}$ such that $\left(I_{1} \cup I_{2}\right) \cap I_{i}=\emptyset$ for $3 \leq i \leq k$, and $I_{1} \cup \cdots \cup I_{k} \in\left(N_{1} \cap N_{2}\right) \cup \mathcal{B}_{\max }$ (note that the family $\left\{I_{3}, \ldots, I_{k}\right\}$ can be empty).

Proof. As before, we proceed by induction on $\operatorname{rk}(\mathcal{B})$. If $\operatorname{rk}(\mathcal{B})=1$ then $I_{1}=\{i\}$ and $I_{2}=\{j\}$, with $\{i, j\}$ the only non-singleton member of $\mathcal{B}$. This makes the properties $(1)-(3)$ obvious.

So we assume that $\operatorname{rk}(\mathcal{B})>1$, choose $C \in N_{1} \cap N_{2}$, and assume that (1) - (3) hold for the building $\mathcal{B}^{\prime}=\left.\mathcal{B}\right|_{C} \times C \backslash \mathcal{B}$ and its maximal nested sets $N_{1}^{\prime}$ and $N_{2}^{\prime}$ corresponding to $N_{1}$ and $N_{2}$ as in Proposition 3.2. Thus, for $i \in\{1,2\}$, we have $N_{i}^{\prime}=\left\{I^{\prime}: I \in N_{i}-\{C\}\right\}$, where the correspondence $I \mapsto I^{\prime}$ is given by (3.7). For the sake of convenience, we refer to the properties (1) - (3) for the sets $N_{1}^{\prime}$ and $N_{2}^{\prime}$ as $\left(1^{\prime}\right)-\left(3^{\prime}\right)$. So we need to show that $\left(1^{\prime}\right)-\left(3^{\prime}\right)$ imply $(1)-(3)$.

First of all, by the definition of $\mathcal{B}^{\prime}$, every $J \in \mathcal{B}^{\prime}$ is contained in either $C$ or $S-C$. Hence $\left(3^{\prime}\right)$ implies that either both $I_{1}^{\prime}$ and $I_{2}^{\prime}$ are contained in $C$ or both are contained in $S-C$. Remembering (3.7), we see that $\left(1^{\prime}\right)$ implies (1).

To prove (2) suppose that $J=I_{1} \cap I_{2} \neq \emptyset$. There are three possibilities to consider:

- $J$ is strictly contained in $C$.

- $J$ contains $C$.

- $J$ is disjoint from $C$.

If $J$ is strictly contained in $C$ then both $I_{1}$ and $I_{2}$ belong to the set $\mathcal{B}_{1}$ in (3.4). By (3.7), we have $I_{i}^{\prime}=I_{i}$ for $i \in\{1,2\}$, hence $I_{1}^{\prime} \cap I_{2}^{\prime}=J$. Again by (3.7), $\left.\mathcal{B}\right|_{J}=\left.\mathcal{B}^{\prime}\right|_{j}$, and so $\left(2^{\prime}\right)$ implies (2).

If $J$ contains $C$ then both $I_{1}$ and $I_{2}$ belong to the set $\mathcal{B}_{2}$ in (3.5). By (3.7), we have $I_{i}^{\prime}=I_{i}-C$ for $i \in\{1,2\}$, hence $J^{\prime}=I_{1}^{\prime} \cap I_{2}^{\prime}=J-C$. If $J^{\prime}=\emptyset$ then $J=C$

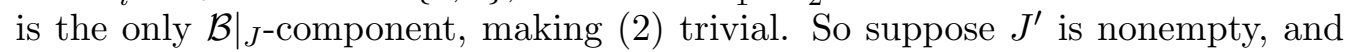
let $J_{1}, \ldots, J_{p}$ be the $\left.\mathcal{B}^{\prime}\right|_{J^{\prime}}$-components. By property (B1) in Definition 2.1 , there can be at most one of the $J_{i}$ such that $C \cup J_{i} \in \mathcal{B}$. If $C \cup J_{i} \notin \mathcal{B}$ for all $i$, then the $\left.\mathcal{B}\right|_{J}$-components are $C, J_{1}, \ldots, J_{p}$; if say $C \cup J_{1} \in \mathcal{B}$, then the $\left.\mathcal{B}\right|_{J}$-components are $C \cup J_{1}, J_{2}, \ldots, J_{p}$. In both cases, (2) follows from $\left(2^{\prime}\right)$, as desired.

It remains to prove (3). Since by Proposition 3.2, every member of $N_{1}^{\prime} \cap N_{2}^{\prime}$ is of the form $I^{\prime}$ for $I \in\left(N_{1} \cap N_{2}\right)-\{C\}$, we assume that $\left(3^{\prime}\right)$ holds with the subsets $I_{3}^{\prime}, \ldots, I_{k}^{\prime} \in N_{1}^{\prime} \cap N_{2}^{\prime}$ in place of $I_{3}, \ldots, I_{k} \in\left(N_{1} \cap N_{2}\right)-\{C\}$.

By the definition of $\mathcal{B}^{\prime},\left(3^{\prime}\right)$ implies that $I_{1}^{\prime}, \ldots, I_{k}^{\prime}$ either are all contained in $C$, or all contained in $S-C$. In the former case, $I_{i}^{\prime}=I_{i}$ for $i=1, \ldots, k$, hence (3) follows at once from $\left(3^{\prime}\right)$. So suppose that $I_{i}^{\prime} \subset S-C$ for $i=1, \ldots, k$. 
Since by $\left(3^{\prime}\right)$ the sets $I_{1}^{\prime}, I_{3}^{\prime}, \ldots, I_{k}^{\prime}$ belong to $N_{1}^{\prime}$, they form a nested set, hence $C \cup I_{i}^{\prime} \in \mathcal{B}$ for at most one index $i \in\{1,3, \ldots, k\}$. By the same token, $C \cup I_{i}^{\prime} \in \mathcal{B}$ for at most one index $i \in\{2,3, \ldots, k\}$. In other words, $I_{i}=C \cup I_{i}^{\prime}$ for at most one index $i$ among $\{1,3, \ldots, k\}$, and at most one among $\{2,3, \ldots, k\}$, while $I_{i}=I_{i}^{\prime}$ for the rest of the indices. Therefore the conditions that $I_{3}, \ldots, I_{k}$ are pairwise disjoint, and that $\left(I_{1} \cup I_{2}\right) \cap I_{i}=\emptyset$ for $3 \leq i \leq k$, follow from the corresponding conditions for the $I_{i}^{\prime}$.

To finish the proof, it remains to consider the following two cases:

- $I_{i}=I_{i}^{\prime}$ for $i=1, \ldots, k$.

- $I_{i}=C \cup I_{i}^{\prime}$ for some $i \in\{1, \ldots, k\}$.

First suppose $I_{i}=I_{i}^{\prime}$ for $i=1, \ldots, k$. Then the condition that $I_{1}^{\prime} \cup \cdots \cup$ $I_{k}^{\prime} \in\left(N_{1}^{\prime} \cap N_{2}^{\prime}\right) \cup \mathcal{B}_{\max }^{\prime}$ means that either $I_{1} \cup \cdots \cup I_{k} \in\left(N_{1} \cap N_{2}\right) \cup \mathcal{B}_{\max }$, or $C \cup I_{1} \cup \cdots \cup I_{k} \in\left(N_{1} \cap N_{2}\right) \cup \mathcal{B}_{\max }$. In the former (resp. latter) case, (3) holds for $I_{3}, \ldots, I_{k}$ (resp. for $I_{3}, \ldots, I_{k}, I_{k+1}=C$ ).

Finally, if $I_{i}=C \cup I_{i}^{\prime}$ for some $i \in\{1, \ldots, k\}$ then $I_{1} \cup \cdots \cup I_{k}=C \cup I_{1}^{\prime} \cup \cdots \cup I_{k}^{\prime} \in$ $\mathcal{B}$ by property (B1) in Definition 2.1. Therefore, $I_{1}^{\prime} \cup \cdots \cup I_{k}^{\prime}=\left(I_{1} \cup \cdots \cup I_{k}\right)^{\prime}$, hence $I_{1} \cup \cdots \cup I_{k} \in\left(N_{1} \cap N_{2}\right) \cup \mathcal{B}_{\max }$, finishing the proof.

Let $D$ be a nested set of cardinality $\operatorname{rk}(\mathcal{B})-2$. As in [8, Section 2.1], we call the induced subgraph of the dual graph whose vertices are the maximal nested sets containing $D$, the geodesic loop associated to $D$. The geodesic loop can be identified with the dual graph of the link of $D$ in $\mathcal{N}(\mathcal{B})$. By a repeated application of Proposition 3.2, a geodesic loop is the dual graph of some complex $\mathcal{N}\left(\mathcal{B}^{\prime}\right)$ with $\operatorname{rk}\left(\mathcal{B}^{\prime}\right)=2$. Thus, by Proposition 4.4, any geodesic loop is a 2-regular graph, hence a disjoint union of cycles. More precisely, we have the following opportunities.

Proposition 4.6. Any geodesic loop is a d-cycle for some $d \in\{3,4,5,6\}$.

Proof. We can assume that $\operatorname{rk}(\mathcal{B})=2$, hence a geodesic loop in question is the dual graph of $\mathcal{N}(\mathcal{B})$. Without affecting this graph, we can assume that there are no singleton $\mathcal{B}$-components of $S$. An easy inspection then leaves us with the following options for $\mathcal{B}$.

(D1) $S=\{1,2,3\} ; \mathcal{B}$ consists of the singletons and $S$. The dual graph is a 3 -cycle with vertices (= maximal nested sets)

$$
\{\{1\},\{2\}\},\{\{2\},\{3\}\},\{\{1\},\{3\}\} .
$$

(D2) $S=\{1,2,3,4\} ; \mathcal{B}$ consists of the singletons, $\{1,3\}$ and $\{2,4\}$. The dual graph is a 4 -cycle with vertices

$$
\{\{1\},\{2\}\},\{\{2\},\{3\}\},\{\{3\},\{4\}\},\{\{1\},\{4\}\} .
$$


(D3) $S=\{1,2,3\} ; \mathcal{B}$ consists of the singletons, $\{1,2\}$, and $S$. The dual graph is a 4 -cycle with vertices

$$
\{\{1\},\{1,2\}\},\{\{2\},\{1,2\}\},\{\{2\},\{3\}\},\{\{1\},\{3\}\} .
$$

(D4) $S=\{1,2,3\} ; \mathcal{B}$ consists of the singletons, $\{1,2\},\{2,3\}$, and $S$. The dual graph is a 5-cycle with vertices

$\{\{1\},\{1,2\}\},\{\{2\},\{1,2\}\},\{\{2\},\{2,3\}\},\{\{3\},\{2,3\}\},\{\{1\},\{3\}\}$.

(D5) $S=\{1,2,3\} ; \mathcal{B}$ consists of all nonempty subsets of $S$. The dual graph is a 6 -cycle with vertices

$\{\{1\},\{1,2\}\},\{\{2\},\{1,2\}\},\{\{2\},\{2,3\}\},\{\{3\},\{2,3\}\},\{\{3\},\{1,3\}\},\{\{1\},\{1,3\}\}$.

We conclude this section by using Proposition 3.2 to obtain a recursion for the $f$-vector of a nested complex. For $k \geq 0$, let $f_{k}(\mathcal{N}(\mathcal{B}))$ denote the number of nested $k$-subsets of $\mathcal{N}(\mathcal{B})$ (so that, in particular, $f_{0}(\mathcal{N}(\mathcal{B}))=1$, and $f_{1}(\mathcal{N}(\mathcal{B}))=$ $\left.|\mathcal{B}|-\left|\mathcal{B}_{\max }\right|\right)$. Let

$$
f(\mathcal{N}(\mathcal{B}))=\sum_{k \geq 0} f_{k}(\mathcal{N}(\mathcal{B})) x^{k}
$$

be the corresponding generating function. The following result is an analogue of [7, Proposition 3.7].

Proposition 4.7. (1) We have $f\left(\mathcal{N}\left(\mathcal{B}_{1} \times \mathcal{B}_{2}\right)\right)=f\left(\mathcal{N}\left(\mathcal{B}_{1}\right)\right) f\left(\mathcal{N}\left(\mathcal{B}_{2}\right)\right)$.

(2) For every $k \geq 1$, we have

$$
k f_{k}(\mathcal{N}(\mathcal{B}))=\sum_{C \in \mathcal{B}-\mathcal{B}_{\max }} f_{k-1}\left(\mathcal{N}\left(\left.\mathcal{B}\right|_{C} \times C \backslash \mathcal{B}\right)\right) .
$$

Equivalently,

$$
\frac{d f(\mathcal{N}(\mathcal{B}))}{d x}=\sum_{C \in \mathcal{B}-\mathcal{B}_{\max }} f\left(\mathcal{N}\left(\left.\mathcal{B}\right|_{C}\right)\right) f(\mathcal{N}(C \backslash \mathcal{B})) .
$$

Proof. Part (1) is immediate from the definitions. To prove (2) count in two different ways the number of pairs $(C, N)$, where $C \in \mathcal{B}-\mathcal{B}_{\max }$, and $N$ is a nested $k$-set containing $C$. On the one hand, each $N$ contributes $k$ to this count, so the number in question is equal to $k f_{k}(\mathcal{N}(\mathcal{B}))$; on the other hand, by Proposition 3.2, each $C$ contributes $f_{k-1}\left(\mathcal{N}\left(\left.\mathcal{B}\right|_{C} \times C \backslash \mathcal{B}\right)\right)$, adding up to the right hand side of (4.1).

It would be interesting to compare the recursion for the $f$-vector given by (4.1) with the one in $[9$, Theorem $7.11(3)]$. 


\section{Nested fans}

We now realize the nested complex $\mathcal{N}(\mathcal{B})$ associated to a building $\mathcal{B}$ on a finite set $S$ as a smooth complete simplicial fan in a real vector space of dimension $\operatorname{rk}(\mathcal{B})$. Let $\mathbb{R}^{S}$ (resp. $\mathbb{Z}^{S}$ ) be a real vector space (resp. a lattice) with the distinguished basis $\left\{e_{i}: i \in S\right\}$. For a subset $I \subset S$, we denote

$$
e_{I}=\sum_{i \in I} e_{i} \in \mathbb{Z}^{S} \subset \mathbb{R}^{S}
$$

Let $V$ denote the quotient space of $\mathbb{R}^{S}$ modulo the linear span of the vectors $e_{C}$ for $C \in \mathcal{B}_{\max }$ (recall that $\mathcal{B}_{\max }$ stands for the set of all $\mathcal{B}$-components of $S$, that is, the maximal elements of $\mathcal{B})$. Thus, $\operatorname{dim} V=\operatorname{rk}(\mathcal{B})$. Let $\pi: \mathbb{R}^{S} \rightarrow V$ denote the projection, and let $L=\pi\left(\mathbb{Z}^{S}\right) \subset V$ be the standard lattice in $V$. We abbreviate $\bar{e}_{I}=\pi\left(e_{I}\right) \in L$.

Theorem 5.1. (1) For every maximal nested set $N$, the vectors $\bar{e}_{I}$ for $I \in N$ form a $\mathbb{Z}$-basis in $L$.

(2) Every vector $v \in L$ has a unique expansion (referred to as the nested expansion) $v=\sum_{I \in \mathcal{B}-\mathcal{B}_{\max }} c_{I} \bar{e}_{I}$ such that all coefficients $c_{I}$ are nonnegative integers, and the set $\left\{I: c_{I}>0\right\}$ is nested.

Proof. (1) It suffices to show that any maximal nested set $N$ satisfies:

The vectors $e_{I}$ for $I \in N \cup \mathcal{B}_{\max }$ form a $\mathbb{Z}$-basis in $\mathbb{Z}^{S}$.

We proceed by induction on $|N|=\operatorname{rk}(\mathcal{B})$ (see Proposition 4.1). If $\operatorname{rk}(\mathcal{B})=0$ then $N$ is empty, and $\mathcal{B}=\mathcal{B}_{\max }$ consists of the singletons, making (5.1) trivial. If $\operatorname{rk}(\mathcal{B})>0$, choose any $C \in N$ and apply the induction assumption to the building $\mathcal{B}^{\prime}=\left.\mathcal{B}\right|_{C} \times C \backslash \mathcal{B}$ on $S$ and the maximal nested set $N^{\prime} \in \mathcal{N}\left(\left.\mathcal{B}\right|_{C} \times C \backslash \mathcal{B}\right)$ corresponding to $N$ as in Proposition 3.2. Replacing $(\mathcal{B}, N)$ with $\left(\mathcal{B}^{\prime}, N^{\prime}\right)$ changes the vectors in (5.1) as follows: for each $I \in N \cup \mathcal{B}_{\max }$ that strictly contains $C$, replace $e_{I}$ with $e_{I-C}=e_{I}-e_{C}$. Clearly, this transformation and its inverse preserve the set of $\mathbb{Z}$-bases in $\mathbb{Z}^{S}$, and we are done.

(2) Any $v \in L$ can be uniquely expressed as $v=\pi\left(\sum_{i \in S} c_{i} e_{i}\right)$, where all $c_{i}$ are nonnegative integers, and $\min _{i \in C} c_{i}=0$ for every $C \in \mathcal{B}_{\max }$. Let $S_{1} \supset S_{2} \supset \cdots$ be the decreasing sequence of subsets of $S$ given by $S_{j}=\left\{i \in S: c_{i} \geq j\right\}$. Then we have

$$
v=\sum_{j \geq 1} \sum_{I \in\left(\left.\mathcal{B}\right|_{S_{j}}\right)_{\max }} \bar{e}_{I}
$$

Collecting similar terms in (5.2) yields an expansion of $v$ into a nonnegative integer linear combination of the $\bar{e}_{I}$ for $I \in \mathcal{B}-\mathcal{B}_{\max }$. It is easy to see that this expansion is nested, i.e., the contributing sets $I$ form a nested set. 
Now let $v=\sum_{I \in \mathcal{B}-\mathcal{B}_{\max }} c_{I} \bar{e}_{I}$ be a nested expansion, and let $N=\left\{I: c_{I}>0\right\}$ be the corresponding nested set. Let $I_{1}, \ldots, I_{k}$ be the maximal (by inclusion) elements of $N$, and let $S_{1}=I_{1} \cup \cdots \cup I_{k}$. As an easy consequence of the definition of a nested set, the sets $I_{1}, \ldots, I_{k}$ are the $\left.\mathcal{B}\right|_{S_{1}}$-components; in particular, we see that $S_{1}$ does not contain any $\mathcal{B}$-component. Applying the same construction to the vector $v^{\prime}=v-\sum_{j=1}^{k} \bar{e}_{I_{j}}$, and continuing in the same manner, we conclude that the nested expansion of $v$ that we started with, is given by (5.2). This proves the uniqueness of a nested expansion, and concludes the proof of Theorem 5.1.

For every nested set $N \in \mathcal{N}(\mathcal{B})$, let $\mathbb{R}_{\geq 0} N$ denote the cone in $V$ generated by the vectors $\bar{e}_{I}$ for $I \in N$. Theorem 5.1 has the following geometric corollary.

Corollary 5.2. The cones $\mathbb{R}_{\geq 0} N$ for $N \in \mathcal{N}(\mathcal{B})$ form a smooth complete simplicial fan in $V$.

We call the fan in Corollary 5.2 the nested fan associated to $\mathcal{B}$, and denote it by $\Delta(\mathcal{B})$. Corollary 5.2 follows from Theorem 5.1 by fairly standard arguments (see e.g., [7, Section 3.4, proof of Theorem 1.10]). For the reader's convenience, here is a self-contained proof.

Proof of Corollary 5.2. By Theorem 5.1 (1), every cone $\mathbb{R}_{\geq 0} N$ for $N \in \mathcal{N}(\mathcal{B})$ is simplicial and is generated by a part of a $\mathbb{Z}$-basis in the lattice $L$; in other words, if these cones form a fan, then this fan is simplicial and smooth. To check that the nested fan is well defined, we need to show that

$$
\mathbb{R}_{\geq 0} N_{1} \cap \mathbb{R}_{\geq 0} N_{2}=\mathbb{R}_{\geq 0}\left(N_{1} \cap N_{2}\right) \quad\left(N_{1}, N_{2} \in \mathcal{N}(\mathcal{B})\right) ;
$$

the completeness property asserts that

$$
\bigcup_{N \in \mathcal{N}(\mathcal{B})} \mathbb{R}_{\geq 0} N=V
$$

Let us first show (5.4). The existence of a nested expansion in Theorem 5.1 (2) means that $\bigcup_{N \in \mathcal{N}(\mathcal{B})} \mathbb{Z}_{\geq 0} N=L$, and so the union $\bigcup_{N \in \mathcal{N}(\mathcal{B})} \mathbb{Q}_{\geq 0} N$ is the $\mathbb{Q}$-vector space generated by $L$. Therefore, the union $\bigcup_{N \in \mathcal{N}(\mathcal{B})} \mathbb{R}_{\geq 0} N$ is dense in $V$; since it is also closed, (5.4) follows.

Turning to (5.3), we need to show that

$$
\mathbb{R}_{\geq 0} N_{1} \cap \mathbb{R}_{\geq 0} N_{2} \subset \mathbb{R}_{\geq 0}\left(N_{1} \cap N_{2}\right)
$$

(the reverse inclusion is trivial). Clearly, it suffices to show the following:

$$
\text { If } N_{1}, N_{2} \in \mathcal{N}(\mathcal{B}) \text { and } \mathbb{R}_{>0} N_{1} \cap \mathbb{R}_{>0} N_{2} \neq \emptyset \text {, then } N_{1}=N_{2} \text {, }
$$

where $\mathbb{R}_{>0} N$ stands for the set of all positive linear combinations of the vectors $\bar{e}_{I}$ for $I \in N$. Let $\mathbb{R} N$ denote the linear span of the cone $\mathbb{R}_{\geq 0} N$. Since each of 
the cones $\mathbb{R}_{\geq 0} N_{1}$ and $\mathbb{R}_{\geq 0} N_{2}$ is generated by a part of a $\mathbb{Z}$-basis in $L$, the vector subspace $\mathbb{R} N_{1} \cap \mathbb{R} N_{2} \subset V$ is defined over $\mathbb{Z}$. The intersection $\mathbb{R}_{>0} N_{1} \cap \mathbb{R}_{>0} N_{2}$ is an open polyhedral cone in $\mathbb{R} N_{1} \cap \mathbb{R} N_{2}$. Therefore, if $\mathbb{R}_{>0} N_{1} \cap \mathbb{R}_{>0} N_{2} \neq \emptyset$ then $\mathbb{Z}_{>0} N_{1} \cap \mathbb{Z}_{>0} N_{2} \neq \emptyset$, and so $N_{1}=N_{2}$ by the uniqueness of a nested expansion in Theorem $5.1(2)$.

As a standard consequence of Corollary 5.2, we get the following corollary.

Corollary 5.3. The dual graph (see Definition 4.3) of any nested complex is connected.

\section{Nested POLYTOPES}

Theorem 6.1. The nested fan $\Delta(\mathcal{B})$ in Corollary 5.2 is the normal fan of a simple convex polytope $\Pi(\mathcal{B})$, which can be explicitly realized as the set of all tuples $\left(x_{i}\right)_{i \in S}$ of real numbers satisfying:

$$
\begin{aligned}
& \sum_{i \in C} x_{i}=0 \quad\left(C \in \mathcal{B}_{\max }\right) \\
& \sum_{i \in I} x_{i} \leq|I|\left(2^{|C|-1}-2^{|I|-1}\right) \quad\left(I \in \mathcal{B}-\mathcal{B}_{\max }, I \subset C \in \mathcal{B}_{\max }\right) .
\end{aligned}
$$

Before proving Theorem 6.1, we recall relevant facts on normal fans (see [11] or $[2$, Section 2.1]).

Definition 6.2. Let $\Pi$ be a full-dimensional simple convex polytope in a real vector space $V^{*}$. The support function of $\Pi$ is a real-valued function $F$ on the dual vector space $V$ given by

$$
F(v)=\max _{\varphi \in \Pi}\langle v, \varphi\rangle .
$$

The normal fan of $\Pi$ is the complete simplicial fan in $V$ whose cones are in the following inclusion-reversing bijection with the faces of $\Pi$ : each face $\Sigma \subset \Pi$ gives rise to the cone

$$
\mathcal{C}_{\Sigma}=\{v \in V:\langle v, \varphi\rangle=F(v) \text { for } \varphi \in \Sigma\},
$$

whose codimension is equal to $\operatorname{dim}(\Sigma)$.

Now let $\Delta$ be a complete simplicial fan in $V$, and let $R$ be a set of representatives of 1-dimensional cones in $\Delta$. The following proposition (cf. [2, Lemma 2.1]) gives a criterion for $\Delta$ to be the normal fan of a simple convex polytope $\Pi \subset V^{*}$.

Proposition 6.3. The following conditions are equivalent:

(1) $\Delta$ is the normal fan of a full-dimensional simple convex polytope $\Pi$ in $V^{*}$. 
(2) There exists a real-valued function $F$ on $R$ satisfying the following system of linear inequalities. For each pair of adjacent maximal cones $\mathcal{C}$ and $\mathcal{C}^{\prime}$ in $\Delta$, let $\{e\}=(R \cap \mathcal{C})-\left(R \cap \mathcal{C}^{\prime}\right)$ and $\left\{e^{\prime}\right\}=\left(R \cap \mathcal{C}^{\prime}\right)-(R \cap \mathcal{C})$, and write the unique (up to a nonzero real multiple) linear dependence between the elements of $R \cap\left(\mathcal{C} \cup \mathcal{C}^{\prime}\right)$ in the form

$$
m_{e} e+m_{e^{\prime}} e^{\prime}-\sum_{v \in R \cap \mathcal{C} \cap \mathcal{C}^{\prime}} m_{v} v=0,
$$

where $m_{e}$ and $m_{e^{\prime}}$ are positive real numbers. Then

$$
m_{e} F(e)+m_{e^{\prime}} F\left(e^{\prime}\right)-\sum_{v \in R \cap \mathcal{C} \cap \mathcal{C}^{\prime}} m_{v} F(v)>0 .
$$

Under these conditions, one can choose the polytope $\Pi$ to be defined by the following system of linear inequalities:

$$
\Pi=\left\{\varphi \in V^{*}:\langle e, \varphi\rangle \leq F(e) \text { for } e \in R\right\} .
$$

Proof of Theorem 6.1. We apply the criterion in Proposition 6.3 to the nested fan $\Delta(\mathcal{B})$. We retain the notation in Section 5. Thus, the ambient space $V$ of $\Delta(\mathcal{B})$ is the quotient space of $\mathbb{R}^{S}$ modulo the linear span of the vectors $e_{C}$ for $C \in \mathcal{B}_{\max }$. Therefore, the dual space $V^{*}$ is naturally identified with the space of real tuples $\left(x_{i}\right)_{i \in S}$ satisfying (6.1). It remains to show that the linear inequalities (6.2) can be identified with those in (6.5), for an appropriate choice of the set of representatives $R$, and the function $F$ on $R$ satisfying (6.4).

By the definition of the nested fan, the set $R \subset V$ in Proposition 6.3 can be chosen as follows: $R=\left\{\bar{e}_{I}: I \in \mathcal{B}-\mathcal{B}_{\max }\right\}$. Applying Proposition 4.5 and using the notation introduced there, we see that every relation of the form (6.3) can be written as follows:

$$
\bar{e}_{I_{1}}+\bar{e}_{I_{2}}-\sum_{J \in\left(\left.\mathcal{B}\right|_{I_{1} \cap I_{2}}\right)_{\max }} \bar{e}_{J}+\sum_{i=3}^{k} \bar{e}_{I_{i}}-\bar{e}_{I_{1} \cup \cdots \cup I_{k}}=0 .
$$

The corresponding inequality (6.4) takes the form

$$
\sum_{i=1}^{k} F\left(\bar{e}_{I_{i}}\right)-\sum_{J \in\left(\left.\mathcal{B}\right|_{I_{1} \cap I_{2}}\right)_{\max }} F\left(\bar{e}_{J}\right)-F\left(\bar{e}_{I_{1} \cup \cdots \cup I_{k}}\right)>0 .
$$

Note that all the sets participating in (6.7) are contained in $I_{1} \cup \cdots \cup I_{k} \in \mathcal{B}$, hence they are contained in the same $\mathcal{B}$-component $C$. Thus, to prove Theorem 6.1 , it suffices to show that (6.7) holds for the function $F$ given by

$$
F\left(\bar{e}_{I}\right)=|I|\left(2^{|C|-1}-2^{|I|-1}\right)
$$

for all participating subsets $I$. In other words, using the abbreviation

$$
f_{n}(m)=m\left(2^{n-1}-2^{m-1}\right),
$$


it is enough to show that, for any positive integer $n$, this function satisfies

$$
\sum_{i=1}^{k} f_{n}\left(p_{i}\right)-\sum_{j=1}^{\ell} f_{n}\left(q_{j}\right)-f_{n}(r)>0
$$

for all nonnegative integers $p_{i}, q_{j}$, and $r$ not exceeding $n$ and such that

$$
\sum_{i=1}^{k} p_{i}-\sum_{j=1}^{\ell} q_{j}-r=0, \quad \sum_{j=1}^{\ell} q_{j}<\min \left(p_{1}, p_{2}\right) .
$$

To prove (6.8), note that, for every positive integers $a$ and $b$, we have (6.10) $f_{n}(a)+f_{n}(b)-f_{n}(a+b)=a\left(2^{a+b-1}-2^{a-1}\right)+b\left(2^{a+b-1}-2^{b-1}\right)>0$. It follows that, under the assumptions (6.9), we have

$$
\sum_{i=3}^{k} f_{n}\left(p_{i}\right)>f_{n}(r)-f_{n}\left(r-\sum_{i=3}^{k} p_{i}\right) ;
$$

therefore, in proving (6.8) we can assume that $k=2$. Again using (6.10), we can also assume that each nonzero $q_{j}$ is equal to 1 . Thus, (6.8) simplifies as follows: (6.11) $f_{n}\left(p_{1}\right)+f_{n}\left(p_{2}\right)-\ell f_{n}(1)-f_{n}(r)>0$ for $p_{1}+p_{2}=\ell+r, \ell<\min \left(p_{1}, p_{2}\right)$, which, after further simplification, reduces to showing that

$$
r 2^{r-1}-p_{1} 2^{p_{1}-1}-p_{2} 2^{p_{2}-1}>0 \text { for } r>\max \left(p_{1}, p_{2}\right) .
$$

To finish the proof, it remains to note that $r 2^{r-1}-p_{1} 2^{p_{1}-1}-p_{2} 2^{p_{2}-1}>\left((r-1) 2^{r-2}-p_{1} 2^{p_{1}-1}\right)+\left((r-1) 2^{r-2}-p_{2} 2^{p_{2}-1}\right) \geq 0$, as required.

In view of Proposition 4.6, Theorem 6.1 has the following corollary.

Proposition 6.4. Every two-dimensional face of the nested polytope is a d-gon for $d \in\{3,4,5,6\}$.

\section{Graphical buildings}

Definition 7.1 ([9], Example 7.2). Let $\Gamma$ be a graph on the set of vertices $S$. Define the graphical building $\mathcal{B}(\Gamma)$ as the set of all nonempty subsets $C \subset S$ of vertices such that the induced subgraph $\left.\Gamma\right|_{C}$ is connected.

A graphical building is indeed a building since it clearly satisfies conditions (B1) and (B2) in Definition 2.1. Remembering Definition 3.1, we obtain the following proposition.

Proposition 7.2. Suppose $\mathcal{B}$ is a graphical building on $S$. 
(1) For any nonempty $C \subset S$, the restriction $\left.\mathcal{B}\right|_{C}$ is graphical.

(2) For any $C \in \mathcal{B}$, the contraction $C \backslash \mathcal{B}$ is graphical.

Proof. Part (1) is clear since $\left.\mathcal{B}\right|_{C}=\mathcal{B}\left(\left.\Gamma\right|_{C}\right)$. To show (2), it is enough to notice that $C \backslash \mathcal{B}=\mathcal{B}\left(\Gamma^{\prime}\right)$, where $\Gamma^{\prime}$ is the graph obtained from the induced subgraph $\left.\Gamma\right|_{S-C}$ by adjoining all edges $\{s, t\}$ such that each of $s$ and $t$ is connected by an edge in $\Gamma$ with some vertex from $C$.

We now provide a characterization of graphical buildings.

Proposition 7.3. For a building $\mathcal{B}$, the following conditions are equivalent:

(1) $\mathcal{B}$ is graphical.

(2) If $J, I_{1}, \ldots, I_{k} \in \mathcal{B}$ are such that $J \cup I_{1} \cup \cdots \cup I_{k} \in \mathcal{B}$, then $J \cup I_{i} \in \mathcal{B}$ for some $i$.

Proof. The implication (1) $\Longrightarrow(2)$ is obvious. To prove $(2) \Longrightarrow(1)$, note that an arbitrary building $\mathcal{B}$ gives rise to a graph $\Gamma$ on the set of vertices $S$, with $s, t \in S$ joined by an edge whenever $\{s, t\} \in \mathcal{B}$. Repeatedly using conditions (B1) and (B2) from Definition 2.1, we obtain the inclusion $\mathcal{B}(\Gamma) \subset \mathcal{B}$. It remains to show that the reverse inclusion $\mathcal{B} \subset \mathcal{B}(\Gamma)$ holds if $\mathcal{B}$ satisfies (2).

Let $C \in \mathcal{B}$. If $p=|C| \leq 2$ then $C \in \mathcal{B}(\Gamma)$ by the definition of $\Gamma$; so suppose that $p>2$. Repeatedly using (2), we conclude that the elements $s_{1}, \ldots, s_{p}$ of $C$ can be ordered in such a way that $\left\{s_{1}, \ldots, s_{k}\right\} \in \mathcal{B}$ for $k=1, \ldots, p$. In particular, using induction on $p$, we can assume that $\left\{s_{1}, \ldots, s_{p-1}\right\} \in \mathcal{B}(\Gamma)$. Again using (2) with $J=\left\{s_{p}\right\}$, and $I_{1}, \ldots, I_{k}$ being the singletons $\left\{s_{1}\right\}, \ldots,\left\{s_{p-1}\right\}$, we see that $s_{p}$ is joined by an edge in $\Gamma$ with some $s_{i}$ for $1 \leq i<p$. It follows that $C \in \mathcal{B}(\Gamma)$, as desired.

We conclude with several results showing that an analogy between the nested complexes and the cluster complexes in $[7,2]$ becomes sharper for graphical buildings.

First, as an immediate consequence of Proposition 7.3, the nested complex of a graphical building has the following "clique" property.

Corollary 7.4. For a graphical building $\mathcal{B}$, a subset $N \subset \mathcal{B}-\mathcal{B}_{\max }$ is nested if and only if any $I, J \in N$ satisfy (2.1).

Second, for graphical buildings the property (3) of Proposition 4.5 can be strengthened as follows.

Corollary 7.5. Suppose $\mathcal{B}$ is graphical, and let $N_{1}$ and $N_{2}$ be two maximal nested sets in $\mathcal{N}(\mathcal{B})$ joined by an edge in the dual graph, so that $N_{1} \cap N_{2}=N_{1}-\left\{I_{1}\right\}=$ $N_{2}-\left\{I_{2}\right\}$. Then, in addition to properties (1) and (2) of Proposition 4.5, we have: $I_{1} \cup I_{2} \in\left(N_{1} \cap N_{2}\right) \cup \mathcal{B}_{\max }$. 
Proof. It suffices to show that, for $\mathcal{B}$ graphical, the family $\left\{I_{3}, \ldots, I_{k}\right\}$ in Proposition 4.5 (3) must be empty. Suppose this is not true, and choose $k \geq 3$ to be the smallest possible. Then $I_{i} \cup I_{j} \notin \mathcal{B}$ for $3 \leq i<j \leq k$ (otherwise, replacing the pair $\left\{I_{i}, I_{j}\right\}$ with one set $I_{i} \cup I_{j}$ would produce a smaller value of $k$ ). Applying the characterization in Proposition 7.3, we obtain that at least one of the sets $I_{1} \cup I_{k}$ and $I_{2} \cup I_{k}$ is in $\mathcal{B}$. But then if, say $I_{1} \cup I_{k} \in \mathcal{B}$ then $I_{1}$ and $I_{k}$ cannot belong to a nested set $N_{1}$, providing the desired contradiction.

Recall that the condition that $N_{1} \cap N_{2}=N_{1}-\left\{I_{1}\right\}=N_{2}-\left\{I_{2}\right\}$ in Corollary 7.5 means that the corresponding cones $\mathbb{R}_{\geq 0} N_{1}$ and $\mathbb{R}_{\geq 0} N_{2}$ are adjacent in the nested fan (see Corollary 5.2).

Corollary 7.6. In the situation of Corollary 7.5, the linear relation (6.6) takes the form

$$
\bar{e}_{I_{1}}+\bar{e}_{I_{2}}-\sum_{J \in\left(\mathcal{B}||_{I_{1} \cap I_{2}}\right)_{\max }} \bar{e}_{J}-\bar{e}_{I_{1} \cup I_{2}}=0 ;
$$

in particular, the vector $\bar{e}_{I_{1}}+\bar{e}_{I_{2}}$ belongs to $\mathbb{R}_{\geq 0}\left(N_{1} \cap N_{2}\right)$.

Finally, for graphical buildings, Proposition 6.4 takes the following stronger form.

Proposition 7.7 ([10], Section 2.8). If $\mathcal{B}$ is graphical then every two-dimensional face of the nested polytope $\Pi(\mathcal{B})$ is a d-gon for $d \in\{4,5,6\}$.

Proof. In view of Propositions 3.2 and 7.2, every two-dimensional face of $\Pi(\mathcal{B})$ corresponds to the nested complex of a graphical building of rank 2. Such complexes were listed in the proof of Proposition 4.6. It remains to observe that the only one among them for which the corresponding nested polytope is a triangle (case (D1)) is non-graphical.

To illustrate the above results, let us compare the nested fans and polytopes associated with rank 2 building sets in cases (D2) and (D3) in the proof of Proposition 4.6. Both cases are shown in Figure 1. In case (D2) the building is graphical, while in (D3) it is not. In the former case, the relations (7.1) take the form

$$
\bar{e}_{1}+\bar{e}_{3}=0=\bar{e}_{2}+\bar{e}_{4},
$$

while in the latter, we have (see (6.6))

$$
\bar{e}_{1}+\bar{e}_{2}=\bar{e}_{12}=-\bar{e}_{3} .
$$

As a geometric consequence, in (D2) the nested polytope is a square, while in (D3) it is a trapezoid.

Much more examples and pictures can be found in [9, Section 8]. 


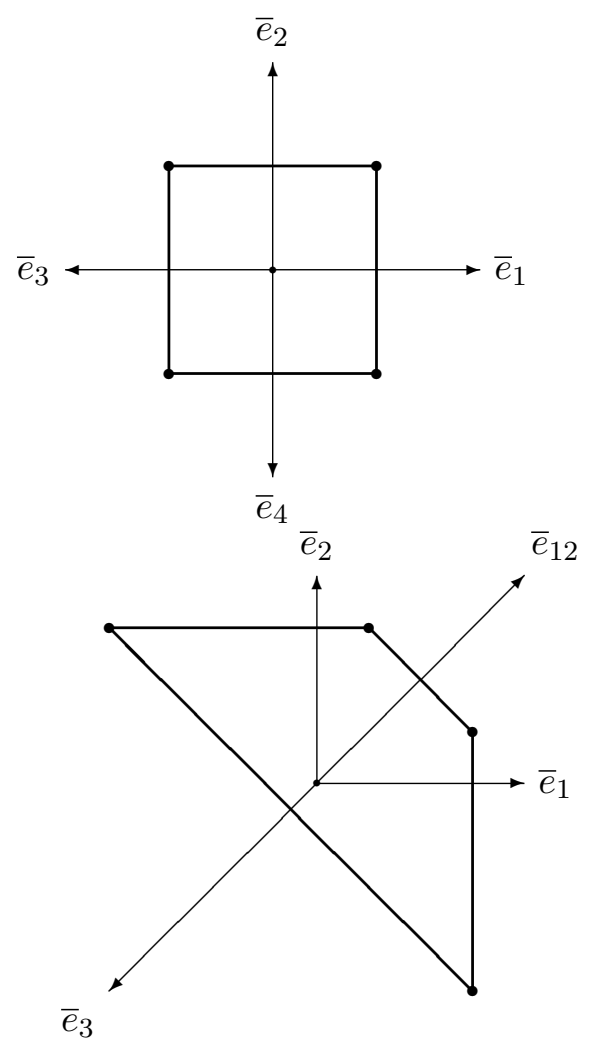

Figure 1. The nested fan and polytope in cases (D2) and (D3).

\section{ACKNOWLEDGMENTS}

I thank Alex Postnikov and Valerio Toledano Laredo for helpful discussions and for sharing with me the ideas and results in their unpublished works. Part of this work was done during my stay at the Mittag-Leffler Institute in May - June 2005; the Institute's hospitality, financial support ant excellent working conditions are gratefully acknowledged.

\section{REFERENCES}

[1] M. Carr and S. Devadoss, Coxeter complexes and graph associahedra, ArXiv: math.QA/0407229.

[2] F. Chapoton, S. Fomin and A. Zelevinsky, Polytopal realizations of generalized associahedra, Canad. Math. Bull. 45 (2002), 537-566.

[3] C. De Concini and C. Procesi, Wonderful models for subspace arrangements, Selecta Math. (N.S.) 1 (1995), 459-494.

[4] E. M. Feichtner and D. N. Kozlov, Incidence combinatorics of resolutions, Selecta Math. (N.S.) 10 (2004), 37-60. 
[5] E. M. Feichtner and B. Sturmfels, Matroid polytopes, nested sets and Bergman fans, Port. Math. (N.S.) 62 (2005), no. 4, 437-468.

[6] E. M. Feichtner and S. Yuzvinsky, Chow rings of toric varieties defined by atomic lattices, Invent. Math. 155 (2004), 515-536.

[7] S. Fomin and A. Zelevinsky, $Y$-systems and generalized associahedra, Ann. in Math. 158 (2003), 977-1018.

[8] S. Fomin and A. Zelevinsky, Cluster algebras II: Finite type classification, Invent. Math. 154 (2003), 63-121.

[9] A. Postnikov, Permutohedra, associahedra, and beyond, ArXiv: math.c0/0507163.

[10] V. Toledano Laredo, Quasi-Coxeter algebras, Dynkin diagram cohomology and quantum Weyl groups, ArXiv: math.QA/0506529.

[11] G. M. Ziegler, Lectures on polytopes, Springer-Verlag, New York, 1995.

Andrei Zelevinsky

Department of Mathematics

Northeastern University

Boston, MA 02115

E-mail: andrei@neu.edu 